\title{
FIRST-PRINCIPLES STUDY OF ELECTRONIC AND MAGNETIC PROPERTIES OF ANATASE AND ITS ROLE IN ANATASE-MXENE NANOCOMPOSITE
}

\author{
D. Parajuli, G. C. Kaphle, K. Samatha
}

Journal of Nepal Physical Society

Volume 5, Issue 1, October 2019

ISSN : 2392-473X

Editors:

Dr. Vinaya Kumar Jha

Dr. Binod Adhikari

Dr. Kapil Adhikari

JNPS, $5(1), 42-53(2019)$

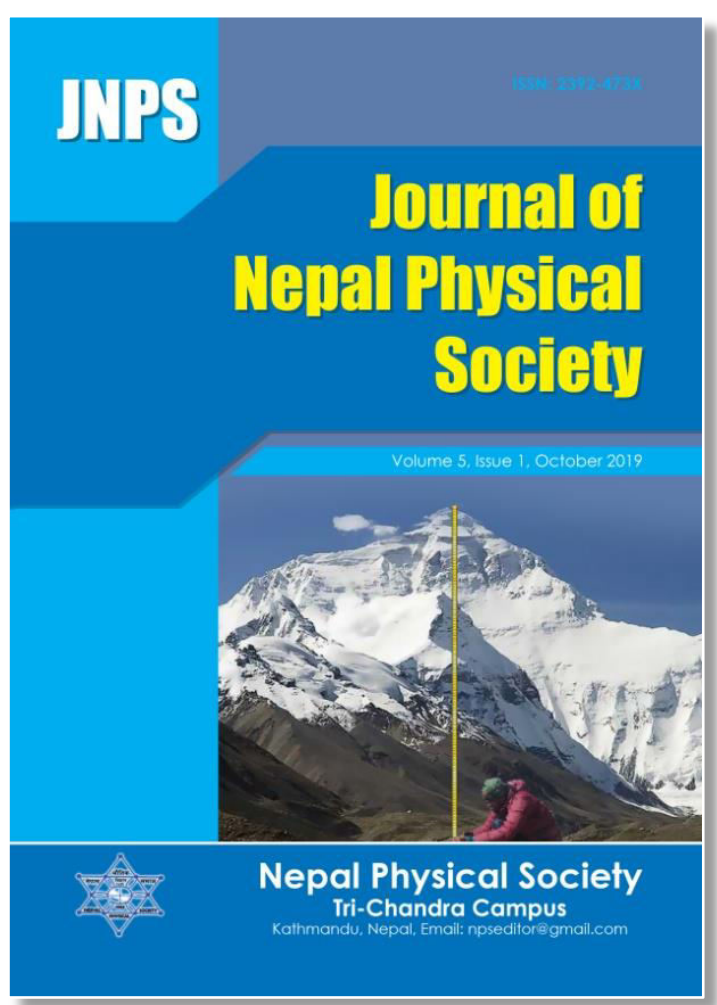

Published by:

Nepal Physical Society

P.O. Box: 2934

Tri-Chandra Campus

Kathmandu, Nepal

Email:npseditor@gmail.com 


\title{
FIRST-PRINCIPLES STUDY OF ELECTRONIC AND MAGNETIC PROPERTIES OF ANATASE AND ITS ROLE IN ANATASE- MXENE NANOCOMPOSITE
}

\author{
D. Parajuli ${ }^{1,2, *}$, G. C. Kaphle ${ }^{3}$, K. Samatha ${ }^{2}$ \\ ${ }^{I}$ Department of Physics, Tri-Chandra Multiple Campus, Ghantaghar, Kathmandu, Nepal \\ ${ }^{2}$ Department of Physics, College of Science \& Technology, Andhra University, Visakhapatnam, India \\ ${ }^{3}$ Central Department of Physics, Tribhuvan University, Kirtipur, Kathmandu, Nepal \\ "Corresponding Email: deepenparaj@gmail.com
}

\begin{abstract}
The electronic and magnetic properties of Titanium and one of its oxide Anatase are calculated by using Tight Binding Linear Muffin-Tin Orbital Atomic Sphere Approximation (TB-LMTO-ASA) method under Density Functional Theory (DFT). The lattice parameter, band structure, Density of States (DOS) and charge density distributions of $\mathrm{Ti}$ and $\mathrm{TiO}_{2}$ (Anatase) required for electronic structure are calculated respectively. The orbital contribution is analyzed by fat band structure; the d- orbital on conduction band and, $s$ and $p$ orbitals on valance bands. Consequently, their magnetic properties are checked. From our study, we found that the magnetic moments of $\mathrm{Ti}$ and $\mathrm{TiO}_{2}$ are found to be $2.2 \mu \mathrm{B}$ and 0 respectively. The total Density of States for spin up and down electron have smaller difference in $\mathrm{Ti}$ and symmetric in $\mathrm{TiO}_{2}$ indicates that $\mathrm{Ti}$ slightly paramagnetic and Anatase is non magnetic in nature. The charge density plots reveals the concentration of electrons at the site under study. Anatase can be deposited onto Mxene to form MxeneAnatase nanocomposite which has several excellent applications in the field of biosensors, biocompatible materials, energy storage devices, topological insulators etc.
\end{abstract}

Key Words: Density Functional Theory, DOS, Band Structures, Photocatalyst, anatase mxene nanocomposites

\section{INTRODUCTION}

Anatase is an oxide of Titanium with two Ti and four oxygen atoms. About $80 \%$ of the world's titanium dioxide is consumed in paints, varnishes, paper and plastics and about $8 \%$ is used in other pigment applications like printing inks, fibers, rubber, cosmetic products and foodstuffs. The rest is used for the production of technical pure titanium, glass and glass ceramics, electrical ceramics, biomedicine photocatalysts, electric conductors, data storage and electronic devices and chemical intermediates [1].

The electronic and magnetic properties of a material depends upon the behavior of valance electrons moving in the field of ion-core of constituent atoms. Titanium (Ti)-22 is a d block transitional metal with hexagonal closed packed (HCP) type of structure and space group P6/mmm and space group number 194 . We have taken value of $\mathrm{c}$ as $4.68 \mathrm{~A}^{\circ}[1]$.

Similarly, Titanium dioxide $\left(\mathrm{TiO}_{2}\right)$ is an important photocatalytic material that exists as two main polymorphs: anatase and rutile. The presence of either or both of these phases impacts on the photocatalytic performance of the material. Titaniumffl dioxide, also known as titania, is of growing interest due to its proven ability to function as a photocatalyst and facilitate important environmentally beneficial reactions, such as water splitting to generate hydrogen and treatment of polluted air and water $[2,3]$.

It is Black, reddish to yellowish brown, dark blue, gray with tetragonal structure. There are 4 atoms per unit cell (Z). It's space group is $\mathrm{I} 4_{1} / \mathrm{amd}$, group number 141 with lattice parameter; $a=3.7845 \AA$, $c=9.5143 \AA$ and $\alpha=\beta=\gamma=90^{\circ}$. It is soluble in HF and insoluble in $\mathrm{H}_{2} \mathrm{O}$. Its hardness is $183 \mathrm{Mohs}$ and Bulk Modulus is $183 \mathrm{GPa}$ [4]. The Atomic Positional Parameters for Ti: 4a; 0.00000 .0000 $\begin{array}{llllll}0.0000 & \text { and } \mathrm{O}: & 8 \mathrm{e} ; & 0.0000 & 0.0000 & 0.2081\end{array}$ respectively. 


\section{MATERIALS AND METHODS}

\section{a. The TBLMTO Approach}

The first principles study of electronic structure calculations in condensed matter have mainly three approaches: 1) Fixed Basis Set Methods: The wavefunction is determined as an expansion in some set of fixed basis functions like linear combination of atomic orbitals (LCAO), plane waves, Gaussian orbitals etc. 2) Partial Wave Methods: The wavefunction is expanded in a set of energy and potential dependent partial waves like the cellular method, the augmented plane wave method and the Korringa-Kohn-Rostoker (KKR) method [5, 6]. 3) LMTO Methods: This method used both of above i. e. it is the linearized version of KKR that combines the desirable features of the fixed basis method and that of partial waves. The potential is assumed to be spherically symmetric close to nuclei or ion core called as muffin tin sphere. The potential is assumed to be flat in between or in interstitial position [7].

\section{b. Muffin-Tin Potential and Partial Waves Solution}

The Density Functional Theory (DFT) is based on the density of electron, the Local Density Approximation (LDA) depend solely upon the value of the electronic density at each point in space and Frozen Core Approximation (FCA) is the consideration of only the valence electron freezing the core electron. The LDA discussed under DFT reduces the many-body Hamiltonian of the valence electron cloud in the presence of a 'frozen' array of ion-cores.

The muffin-tin approximation is a shape approximation of the potential field in an atomistic environment. It is most commonly employed in quantum mechanical simulations of electronic band structure in solids[8]. The muffin tin potential can be defined by:

$\mathrm{V}(\mathrm{r})=\sum_{\mathrm{R}} \mathrm{V}(\mathrm{r}-\mathrm{R})$

where, $\mathrm{R}$ and $\mathrm{r}$ are the position of the ion-cores and the position of the electron as shown in figure 1 .

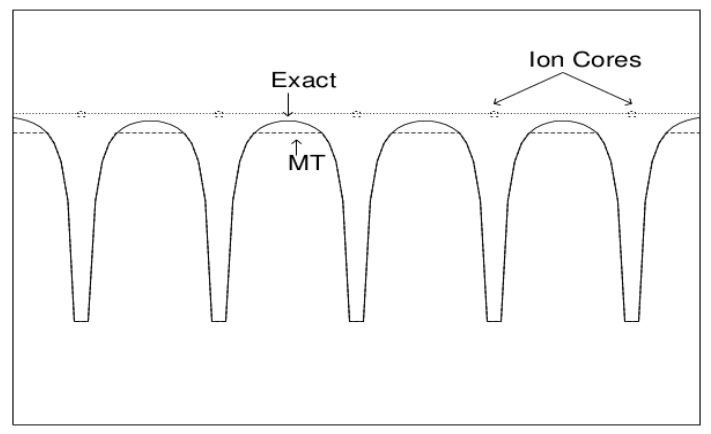

Fig. 1: Construction of muffin-tin potential.

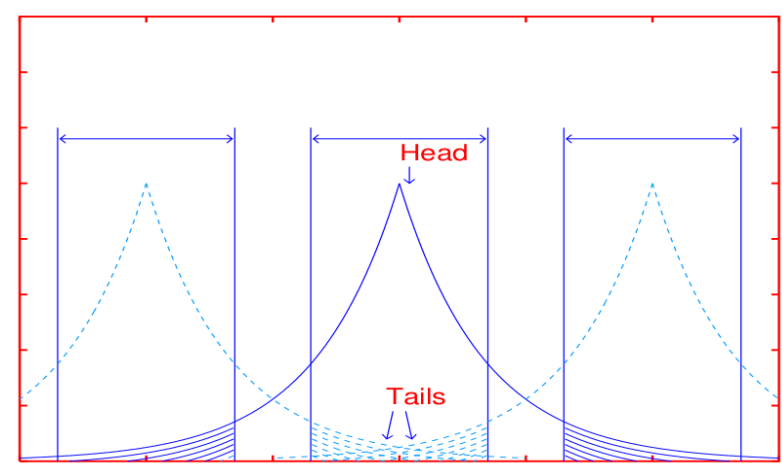

Fig. 2: Tail cancellation.

Before starting full problem of an electron in a solid, we define muffin-tin potential for a single electron ' $\mathrm{e}$ ' inside a single muffin-tin sphere as [9]:

$$
\begin{aligned}
v(r-R)= & v\left(r_{R}\right), \text { for } r_{R} \leq s_{R} \\
& -v_{0}, \text { for } r_{R}>s_{R}
\end{aligned}
$$

where $r_{R}=|r-R|, s_{R}$ is the radius around $R$ in which the potential is spherically symmetric and is called muffin-tin sphere. $\mathrm{s}_{\mathrm{R}}$ is so small that the spheres do not overlap. $-\mathrm{v}_{\mathrm{o}}$ is the slowly varying potential in the interstitial that is considered as constant average. $\mathrm{L}$ is angular momentum levels $(l, m), i^{l}$ is convenient definition, $Y_{L}(r)$ are spherical harmonic functions, $r_{R}$ angular variables associated with the unit vector $(r-R) / r_{R}$ and $\varphi_{R L}\left(r_{R}, \varepsilon\right)$ are solutions of radial equation in the spherical region. The Schrödinger equation for single-electron wavefunction is given by:

$\left[-\nabla^{2}+\mathrm{v}(\mathrm{r}-\mathrm{R})\right] \varphi(\mathrm{r}-\mathrm{R}, \varepsilon)=\varepsilon \varphi(\mathrm{r}-\mathrm{R}, \varepsilon) \cdots$

We can easily solve equation (3) in the spherically symmetric potential as

$\varphi(r-R)=\varphi_{R L}\left(r_{R}, \varepsilon\right) i^{l} Y_{L}(r)$

For $r_{R} \leq s_{R}$ potential is spherically symmetric with solution containing spherical harmonic function $Y_{L}(r)$ and for $\mathrm{r}_{\mathrm{R}}>\mathrm{s}_{\mathrm{R}}$ potential is spherically symmetric with spherical harmonic function $\mathrm{Y}_{\mathrm{L}}(\mathrm{r})$ with radial equation having two linearly independent solutions with Spherical Bessel $j_{l}(k r)$ and Neumann $\eta_{l}(k r)$ functions where $\kappa^{2}=\varepsilon-v_{o}$ gives the kinetic energy of this interstitial region. It is follows that only is regular at origin and both are regular at infinity. As $\mathrm{r}=\mathrm{R}$ is not included in this region, both solutions are allowed and the full solution in this region is the linear combination: $A_{R l}(\varepsilon, \kappa) j_{l}\left(\kappa r_{R}\right)+B_{R l}(\varepsilon, \kappa) n_{l}\left(\kappa r_{R}\right)$ where constants A and $\mathrm{B}$ are determined by applying the boundary condition on the muffin-tin sphere at which both the 
wave function and its derivative are continuous. The part of the partial wave within its muffin-tin or personal sphere centered at $\mathrm{R}$ is called head while outside is called its tail.

\section{c. Muffin-Tin Orbitals, Tail Cancellation and Linearization for Atomic Sphere Approximation}

Head of partial wave contains most of the information about the muffin-tin potential and the tail about the constant potential outside. The tail of the partial wave depends on energy through the phase shift. If $\kappa^{2}$ is negative, it diverges exponentially and is called muffin-tin orbital [1]. The tail has negligible contribution. As a result, the tail from positive direction and negative direction can be canceled as shown in fig 2 . The interstitial positions have such high symmetry that both the muffin-tin and the repulsive interstitial potentials can be approximated spherically symmetric. The interstitial sphere is called 'empty sphere' that form a closed pack structure with muffin-tin sphere[10]. Hence, it is termed as atomic sphere approximation (ASA). The KKR equation is then linearized to get TB-KKR secular equation that leads to the TBLMTO method [11].

\section{d. Self Consistent Calculations}

The calculations of energy minimization, band structure and density of states have been carried out within the framework of density functional theory (DFT). The exchange correlation energy $\mathrm{E}_{\mathrm{xc}}$ have been treated under local density approximation (LDA) [12]. The crystal potential is constructed of overlapping Wigner-Seitz spheres for each atom in the unit cell. In TB-LMTO-ASA procedure, frozen core approximation is included in the selfconsistent calculations of the effective crystal potential [7, 9, 13]. In self consistent calculation, Kohn Sham Equation uses DFT as effective single body problem. To solve this equation, the local density approximation is made and give the initial density of the system to many-body problem to get effective mean field (EMF). The EMF is placed in KS equation. We obtain energy level and corresponding wave function and hence a new electron density of the system. If the electron density is not same as that of initial, they are mixed together and the process is repeated until the difference between initial and final is negligible i. e. The calculations were iterated to self-consistency with an error in total energy less that $10^{-6}$ Rydberg. This is called self consistent procedure and the corresponding field is called self consistent field (scf) $[6,8,14,15,16]$.

\section{e. The computational details}

The structural parameters of the material under study is selected. The lattice parameter and positions are found for which we use directly experimental value for elements and Vegard's law for binary alloys [17]. The final lattice parameter are found by energy minimization method. After the Hartree Fock calculations, the volume of the space is optimized excluding the empty spheres by expanding the oval inside muffin tin spheres. We then run self consistency code which provides all the necessary data to calculate the Band structure, Density of states and Charge density in our system [18]. With the values of optimized lattice parameters, we calculate the electronic nature of s, p, d and f orbitals as band structure and density of state. Finally, we use code to find out the charge density distribution. The syntax of the operation is as follows:

lminit.run: generate a CTRL file with the structural data (i. e. given input) $\rightarrow$ lmhart.run: generate overlapping potentials from atomic Hartree potentials or to find MT-radii $\rightarrow$ lmovl.run: calculate and to display sphere overlaps $\rightarrow$ lmes.run: find interstitial spheres $\rightarrow$ lmctl.run: rewrite the CTRL file according to the parameters in the original CTRL and atomic files and to insert default value $\rightarrow$ lm.run: perform self-consistent iterations $\rightarrow$ lmbnd.run: generate bands for plotting $\rightarrow$ lmdos.run: generate density of states for plotting $\rightarrow$ gnubnd.run: produce data files for energy bands plot $\rightarrow$ gnudos.run: produce data files for density of states plot $[1,7,19,20,21,22]$.

\section{RESULTS AND DISCUSSION}

This includes the energy minimization with optimizing the lattice parameter, band structure, fatband structure of d orbitals, density of states and charge distribution for the electronic structure of $\mathrm{Ti}$ and its oxide (Anatase) for the determination of their electronic and magnetic properties.

\section{a. Energy minimization}

With the use of given lattice parameter, we have optimized the parameter at minimum energy. We have plotted the energy by varying the lattice constant within a certain range where the selfconsistent calculations converged. The graph so obtained was fitted with a second degree polynomial such that there was a minimum value of energy corresponding to a lattice parameter. 


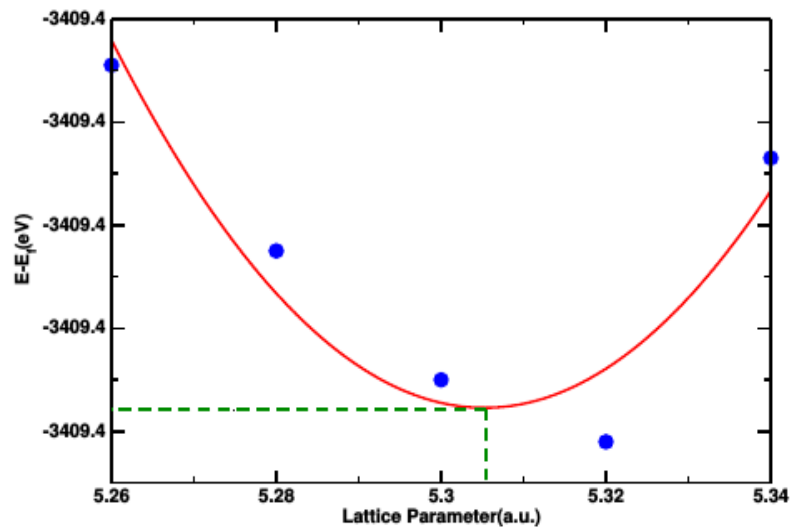

Fig. 3a: Energy Vs Lattice Parameter of Ti.

The circles in the graph between energy and lattice parameter show the actual data points, and the curve represents the best fit. Fig. 3., shows that the value of energy decreases at first, becomes minimum and again starts increasing.

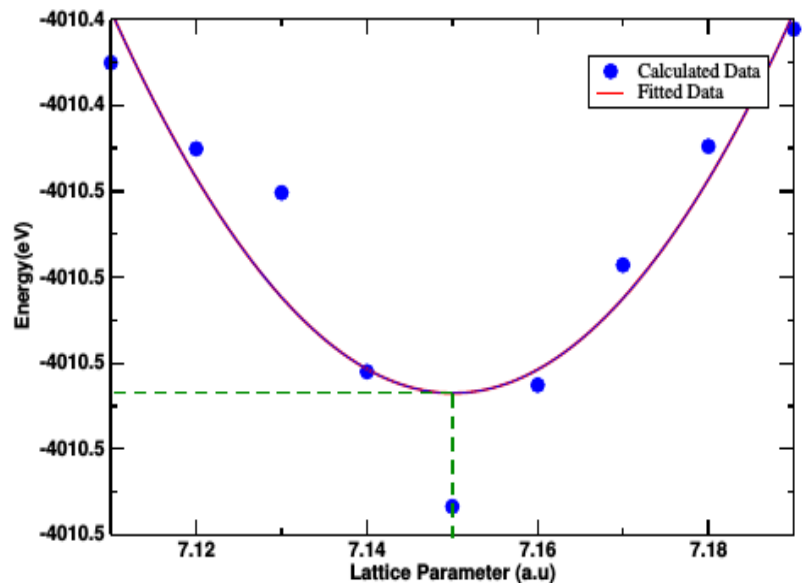

Fig. 3b: Energy Vs Lattice Parameter of $\mathrm{TiO}_{2}$.

The lattice parameter of $\mathrm{Ti}$ at minimum energy is $2.81 \mathrm{~A}^{\circ}(5.31$ a. u) as shown in Fig.3a. The optimized lattice parameter of anatase using Vegards law is 7.149 a.u. Taking this as base value, the optimized lattice parameter is 7.15 a.u. as shown in Fig. 3b.

\section{b. Band Structure Calculations \\ i) Band Structure of Titanium}

The overlapping of valance band and conduction band confirms the metallic nature of Titanium. The two bands lying well below the Fermi level are due to $4 \mathrm{~s}^{2}$ electrons and the bands near the Fermi energy are due to $3 \mathrm{~d}^{2}$ electrons of Titanium. The band structure of titanium from our study is as shown in fig which is in close agreement with the study carried out by G. Glench in 2014 as shown in fig below:

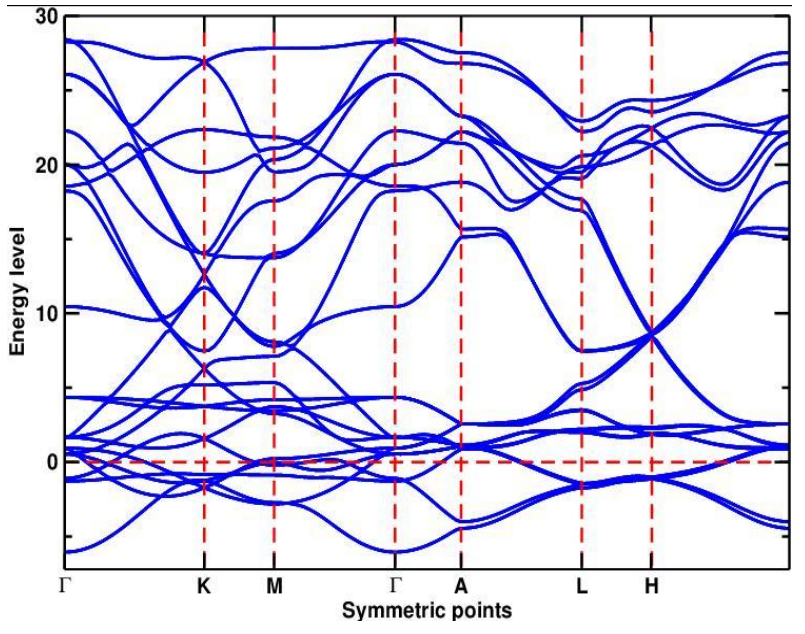

Fig. 4a: Band Structure of Titanium (our plot).

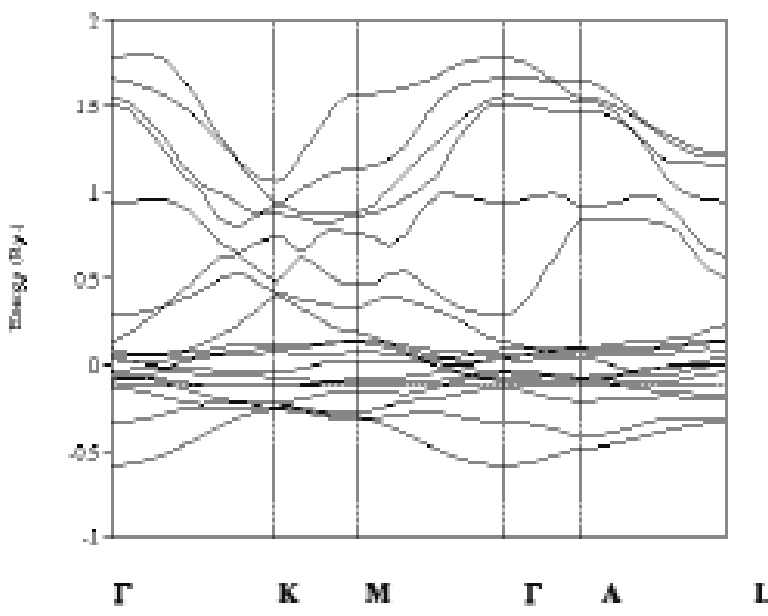

Fig. 4b: Band Structure of Titanium [23].

\section{ii) Fat Band Structures of d orbitals}

The contribution of orbital electrons to the conductivity is mostly due the d orbital electrons as shown from its fat band structure as below [24]:

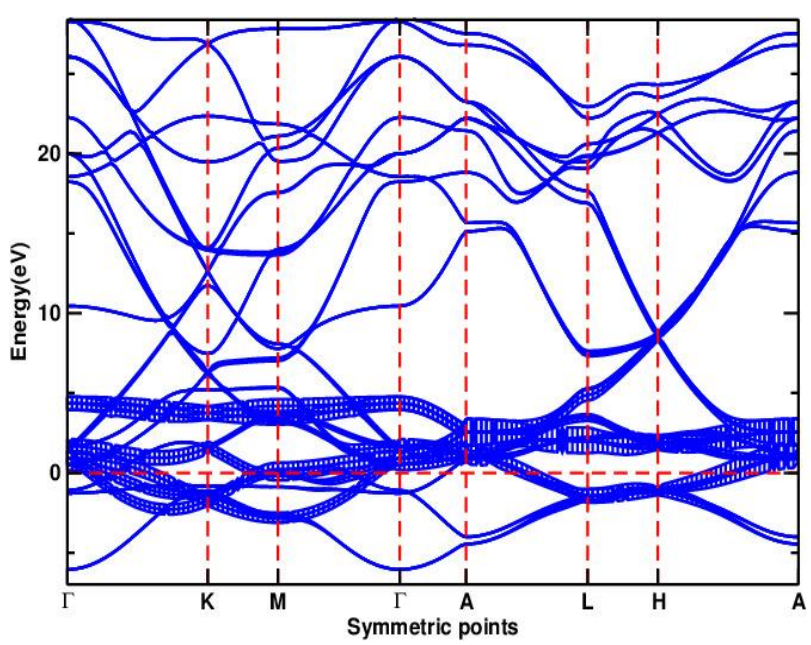

Fig. 5a: $e_{g}$ band Structure of Titanium. 


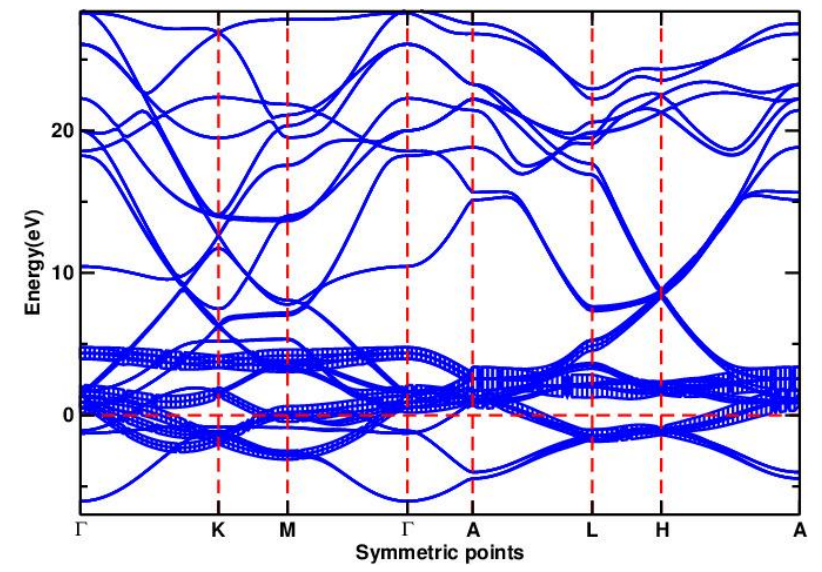

Fig.5b: $t_{2} g$ band Structure of Titanium.

From the Fat Band Structure as shown in Fig. 5 a) and $5 \mathrm{~b}$ ), it is clear that the occupancy of the $d$ band electrons is more near the Fermi energy level showing significant contribution in electronic and magnetic of Titanium. The overlapping between conduction band and valence band indicates the metallic of Titanium.

\section{ii) Band Structure of Anatase}

Fig. 6 a) (not in same scale) below shows the band structure of pure anatase $\mathrm{TiO}_{2}$. It is observed that the band gap is about $2.1 \mathrm{eV}$ is nearly in agreement with the result of $2.21 \mathrm{eV}$ in Fig. $6 \mathrm{~b}$ ) obtained by $\mathrm{Wu}$ et. al. in 2008 using a plane wave pseudopotential method in the framework of the density functional theory and the generalized gradient approximation.

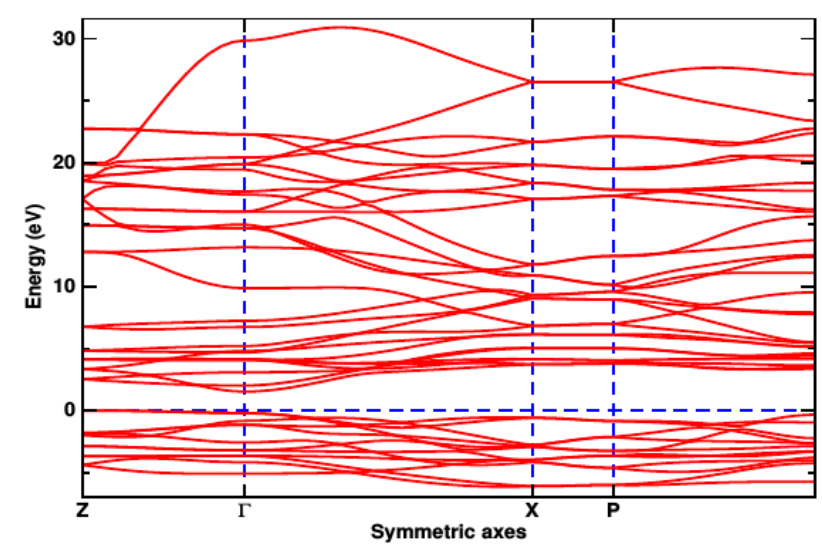

Fig. 6a: Energy band structure of anatase.

The valence band of pure $\mathrm{TiO}_{2}$ mainly consists of the $2 p, 2$ s states of $\mathrm{O}$ and $3 \mathrm{~d}$ states of Ti. In the uppermost valence band, the $\mathrm{O} 2 \mathrm{p}$ states are predominantly found between -5 and $0 \mathrm{eV}$, while the $\mathrm{O} 2 \mathrm{~s}$ states appear in the range from -18 to $15.5 \mathrm{eV}$. The Ti $3 \mathrm{~d}$ states give rise to some bands in the energy range from -5 to $-3 \mathrm{eV}$. The lowest conduction band is dominated by Ti $3 \mathrm{~d}$ states.

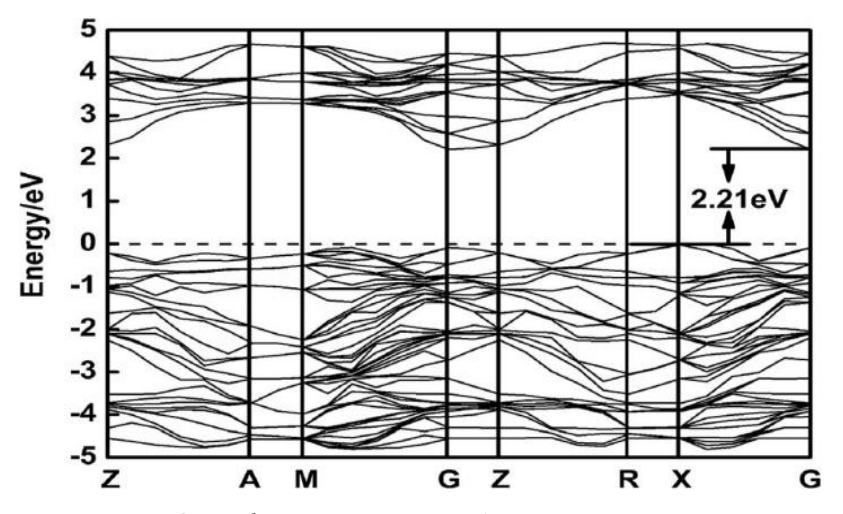

Fig. 6b: Energy Band Structure of Pure anatase [25].

Fig. 6.a) shows that, the energy is minimum at valence band and maximum in conduction bands at gamma point $(\Gamma)$. The band gap at the gamma point from our calculation is $2.10 \mathrm{eV}$ which close to the value $2.21 \mathrm{eV}$ calculated by $\mathrm{Wu}$ et. al. [27] as shown in Fig.6b above.

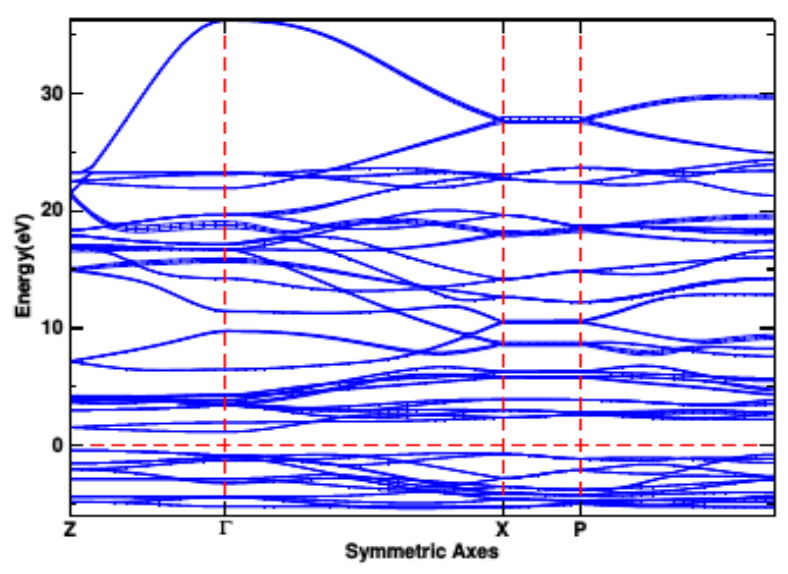

Fig. 7a: Fat band structure of s orbital of anatase.

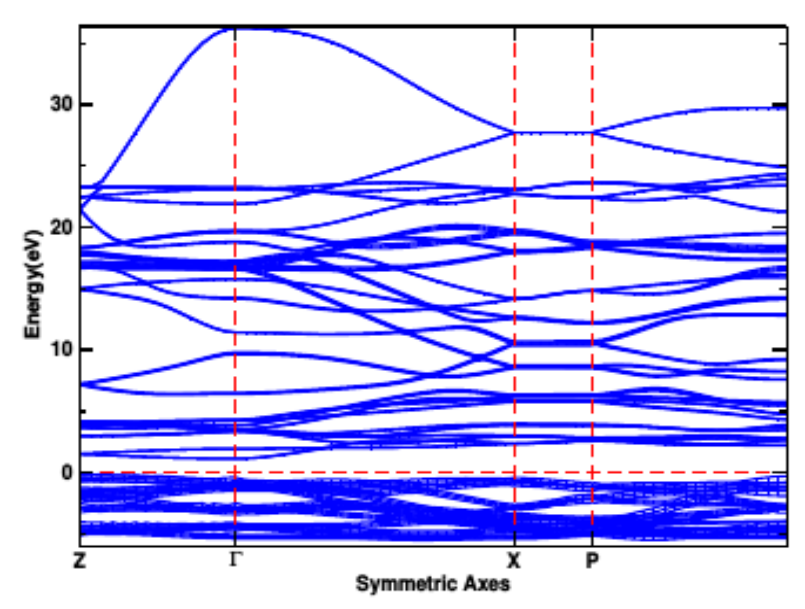

Fig. 7b: Fat band structure of $p$ orbital of anatase. 


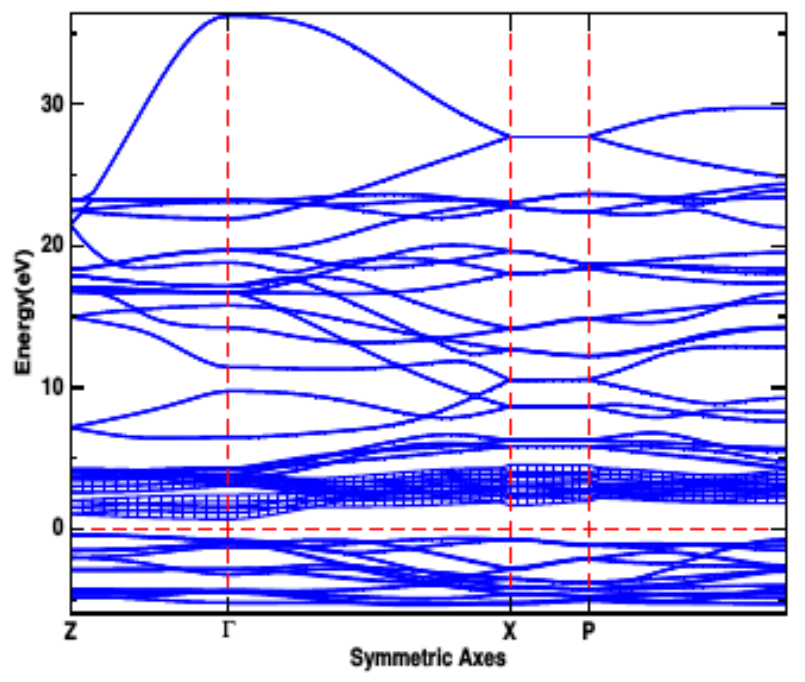

Fig. 8a: Fat band structure of $t_{2} g$ orbital of anatase.

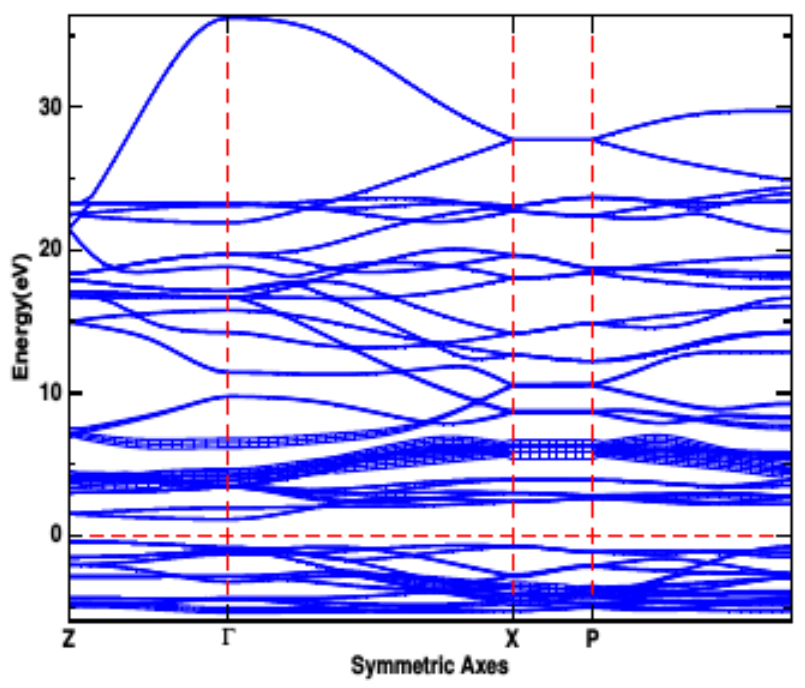

Fig. 8b: Fat band structure of $e_{g}$ orbital of anatase.

We further calculated the fatband structure of anatase with $\mathrm{s}$ and $\mathrm{p}$ orbital characteristics as shown in Fig 7. a) and 7. b). In the Fig 7. a), maximum occupancy of the s electrons is far above the Fermi energy along $\Gamma$, and $\mathrm{X} \& \mathrm{P}$ axis. The Fig.7. b) shows that $\mathrm{p}$ electrons are largely distributed below the Fermi energy level. As a result, the $s$ and $p$ orbital have no contribution in conduction of electron between conduction and valence band.

We also carried out the band structure calculation for the $d$ orbital of anatase showing $t_{2} g$ and $e_{g}$ orbital characteristics as shown in Fig.8.a) and b) respectively which shows that the number of electrons occupying the energy states above the Fermi level in case of $t_{2} g$ orbital is higher than that of $e_{g}$ orbital near the Fermi level but not overlapping the conduction and valence band. So, they also do not have contribution in the physical properties of the solid like electrical and magnetic properties is negligible.

\section{b. Density of States(DOS) Calculations}

DOS helps us to calculate various properties such as internal energy, density of particles, specific heat capacity, magnetic moment and thermal conductivity. The product of density of states and the probability distribution function is the number of occupied states per unit volume at a given energy for a system in thermal equilibrium. Some worth noting points about DOS are: a) DOS of orbital are symmetric about Fermi level have no contribution in magnetic moment, while, the DOS of orbitals anti-symmetric do have. b) The peaks in the DOS signifies the large number of states at the corresponding energy. c) The charge found in any orbital indicates that its contribution in magnetic moment is due to the orbital.

The magnetic moment is obtained by integrating difference of density of states between up and down spin states upto Fermi Energy [26] by computational approach. The projected density of states PDOS have less information than total density of states(TDOS). LDOS stands for the local density of states related to LDA.

\section{i) Density of States of Titanium}

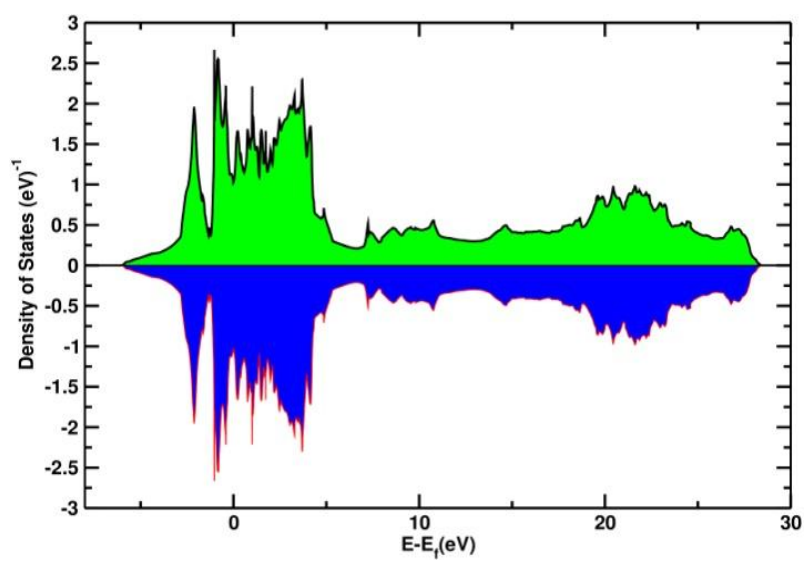

Fig.9a: Total SPDOS of s orbital electrons of Ti.

From the calculated data, the spin projected density of state of Titanium is plotted with reference to the Fermi energy in Fig. 9. The vertical line at zero in the figure represents Fermi energy of the system. Density of states plots above the horizontal axis are due to up spin and below due to down spin states. The region left to the vertical reference line 
indicates valance band and that to the line is conduction band. The total spin projected density of states of Titanium for $s, p$ and d orbital electrons are as shown in Fig.9a, 9b and 9c respectively.

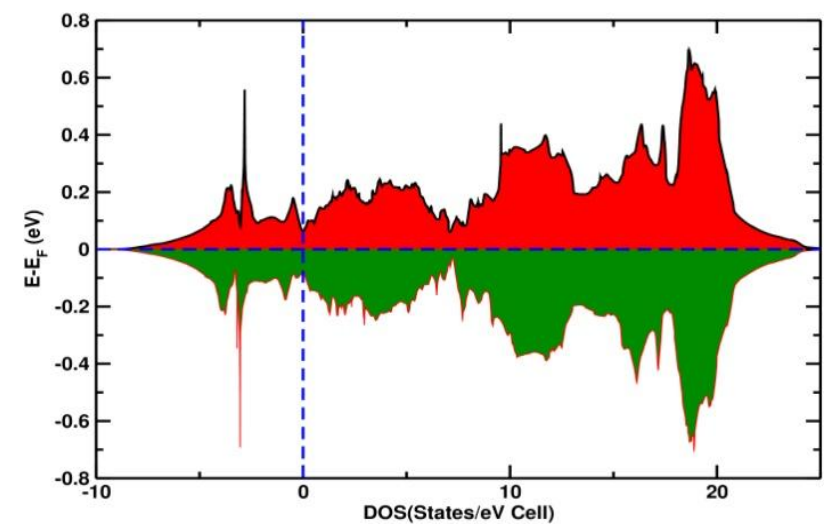

Fig.9b: Total SP DOS of p orbital electrons of Ti.

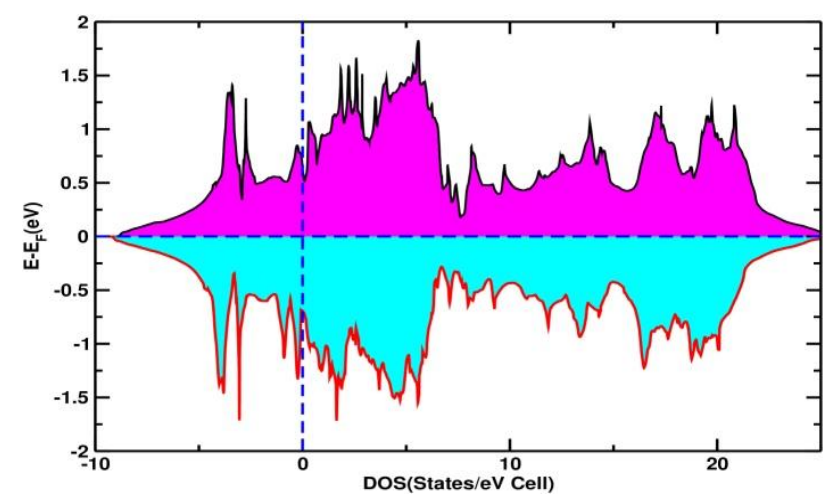

Fig.9c: Total SPDOS of d orbital electrons of Ti.

The combined effect of all the above DOS plots of s, p, d orbitals are as in Fig.10 (not in scale) below. It is clear that the electrons of $\mathrm{d}$ orbitals near the Fermi level have the significant role for conduction which ultimately give the corresponding electronic and magnetic property of Titanium. The DOS plot is in well agreement with the DOS plot made by Gench in 2014.

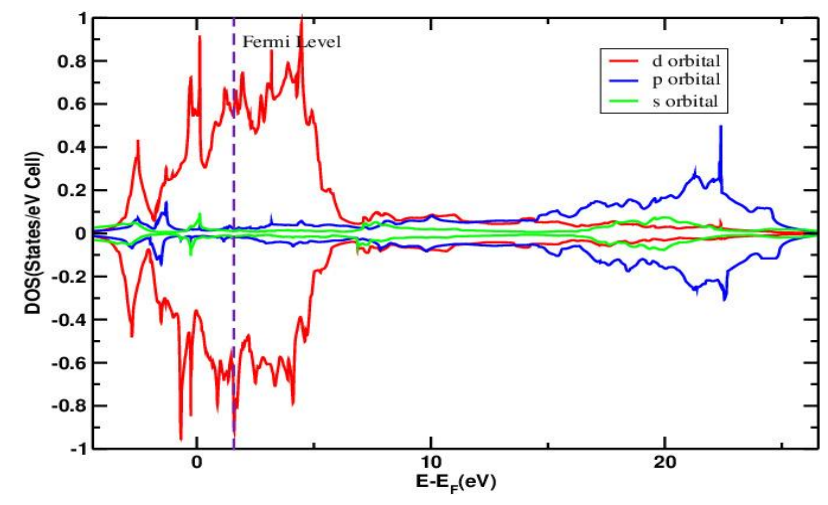

Fig.10a: Color line: Total (spd) DOS of Titanium.

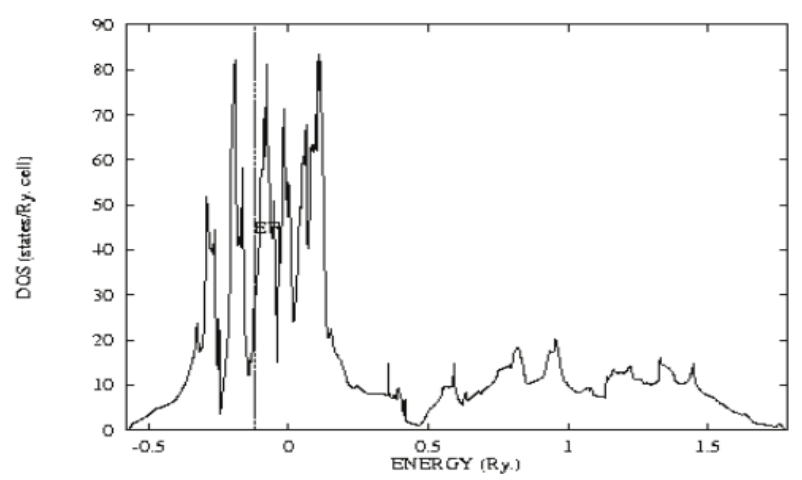

Fig.10b: Color line:DOS (up spin) of Titanium [23].

In the Fig. 10. a), the green line represents the DOS plot of s orbital electron, blue line represents $p$ orbitals electrons and red line represents the $d$ orbital electrons. For $\mathrm{s}$ and $\mathrm{p}$ orbital dos plots, all peaks of density of states of up spin states and down states are lying below the Fermi energy while that of d orbital lie across the Fermi level showing that $d$ orbitals contribute a lot in the electronic and magnetic property of Titanium. Up spins and down peaks of spins density of states are slight difference with each other. As a result, Titanium has some magnetic moment. The magnetic moment obtained by integrating difference between the density of states of up spin and down spin of $\mathrm{Ti}$ is found to be $2.2 \mu \mathrm{B}$. So, Ti is metallic but paramagnetic (slight magnetic) in nature. This value is in close agreement with the value obtained by Glench in 2014 .

\section{i) Density of States of Titanium}

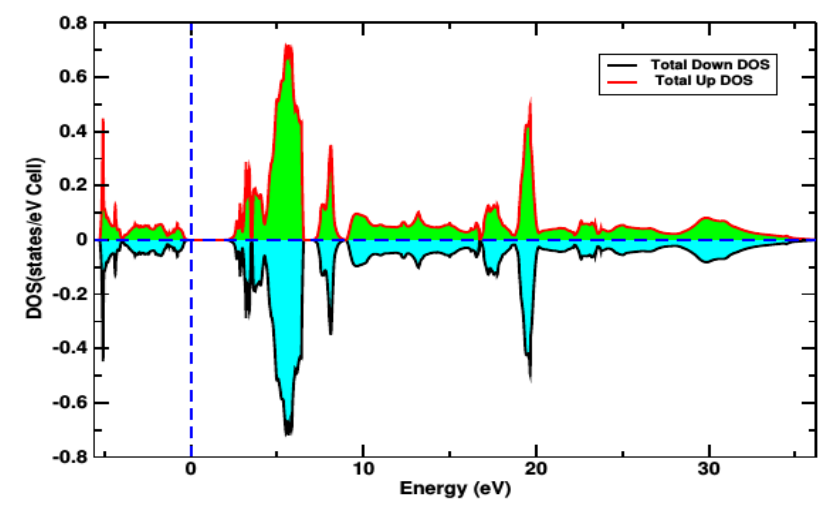

Fig.10a: Spin Projected DOS of s orbital of anatase.

From the calculated data, the total spin projected density of states of anatase for $s, p$ and d orbital electrons are as shown in Fig. 10 a), 10 b) and 10 c) respectively. 


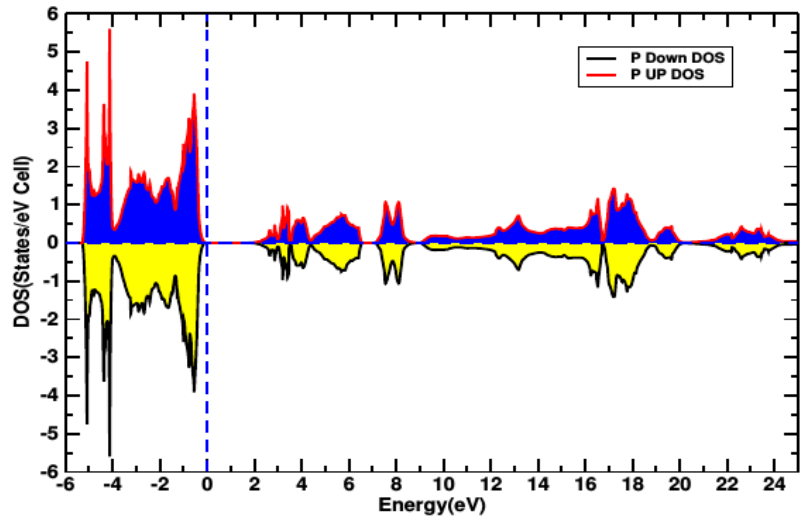

Fig.10b: Spin Projected DOS of p orbital of anatase.

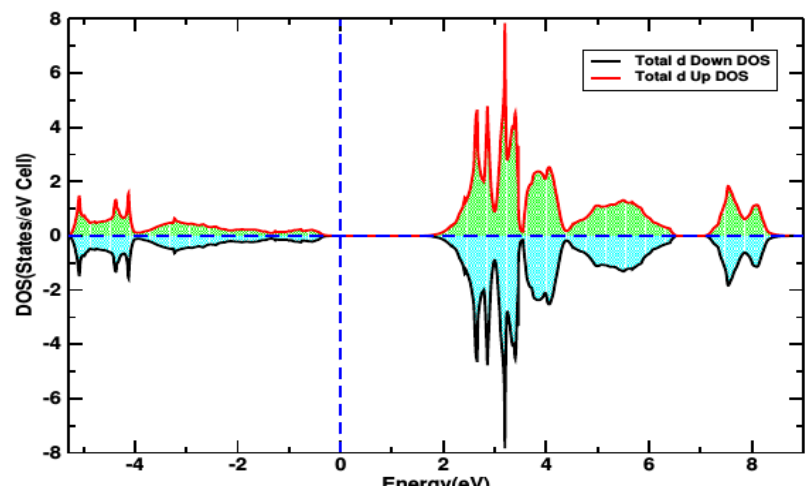

Fig.10c: Spin Projected DOS of d orbital of anatase.

The combined (spd) SPDOS plot of anatase is shown in Fig. 11. a) below. It is clear that the electrons of $d$ orbitals near the Fermi level have the significant role for conduction which ultimately give the corresponding electronic and magnetic property of Titanium. The DOS plot is in well agreement with the DOS plot made by Glench in 2014.

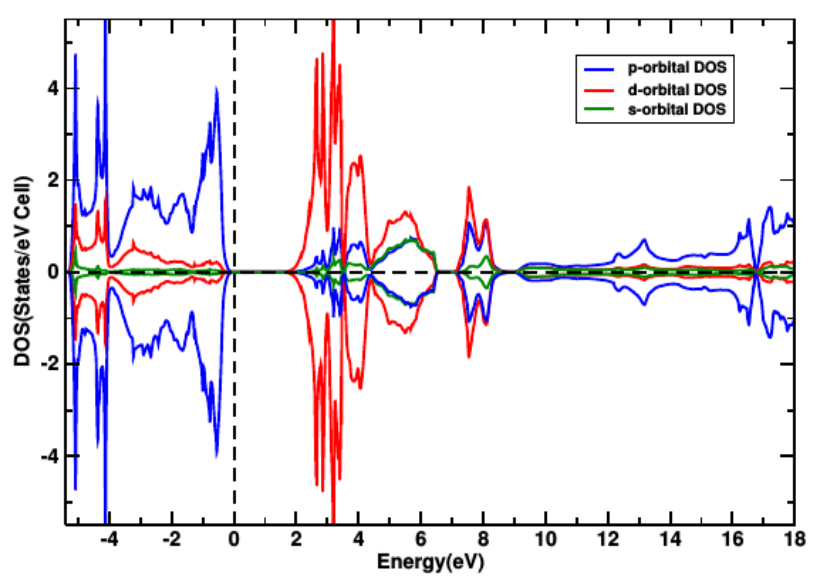

Fig.11a: Combined(spd) SPDOS plot of anatase.
PROJECTED AND TOTAL DENSITY OF STATES

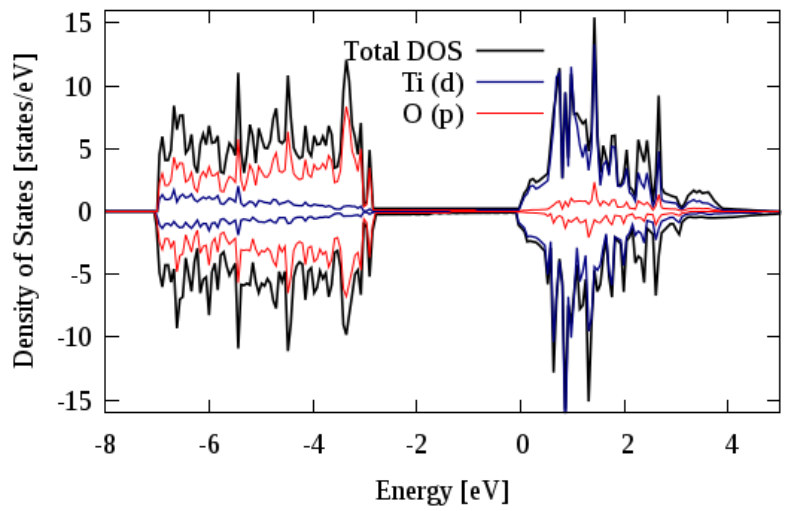

Fig.11b: Combined (spd) SPDOS plot of anatase [27].

In the up and down DOS plot of anatase as shown in Fig. 11. a), the highest peak of up spins lies above the Fermi energy and that of down spins is below the Fermi energy. Also the number of peaks above and below the Fermi level are same in one line i. e. symmetric. From data obtained for density of states of anatase, the magnetic moments of anatase is found to be $0.00000833 \mu \mathrm{B}$, which is negligible at absolute zero. So, anatase has no magnetic moment. This is in agreement with the result recently found by the research group of García in 2018 as shown in Fig. 11. b) above.

\section{e. Charge Distribution Calculations i) Charge density at different orbitals}

The charge density at different orbitals of $\mathrm{Ti}$ are mentioned in the following Table 1.

Table 1: Charge density at different orbitals of Ti.

\begin{tabular}{|c|c|c|c|c|c|c|}
\hline 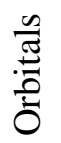 & $\mathrm{S} \uparrow$ & $\mathrm{S} \downarrow$ & $\mathrm{P} \uparrow$ & $\mathrm{P} \downarrow$ & $\mathrm{d} \uparrow$ & $\mathrm{d} \downarrow$ \\
\hline 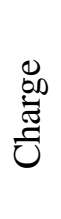 & 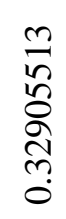 & 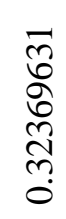 & 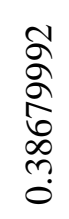 & 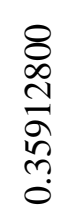 & 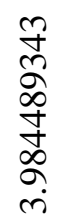 & 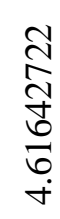 \\
\hline
\end{tabular}

From the charge distribution tables, we observed that there is slightly difference of charges between up spin and down spin state of d-orbital for Ti. But, the contribution of $\mathrm{s}$ and $\mathrm{p}$ orbital in comparison to the $\mathrm{d}$ orbital is negligible. 
The charges are not transferred in any orbital in case of anatase.

\section{f. Charge density at different sites}

It is a measure of electric charge per unit volume of space in one two or three dimensions. Charge density depends on the position as action of point in electrostatic. The value of charge density with respect to the color is shown in charge density scale in fig 12 a). Fig 12 b) shows that the charge distribution in $\mathrm{Ti}$ atom on 001 site where the maximum distribution of the charges occur on the corners. Similarly, Fig 12 c) shows the charge density of anatase in 000 site where the maximum charges occurs at interstitial site and have no contribution in conduction.

\begin{tabular}{|cc|}
\hline \multicolumn{2}{|c|}{ Scale: $\Delta \mathrm{n}(\mathrm{r})$} \\
\hline$\square$ & +1.0000 \\
$\square$ & +2.0000 \\
$\square$ & +3.0000 \\
$\square$ & +4.0000 \\
$\square$ & +5.0000 \\
$\square$ & +6.0000 \\
$\square$ & +7.0000 \\
$\square$ & +8.0000 \\
$\square$ & +9.0000 \\
$\square$ & +10.0000
\end{tabular}
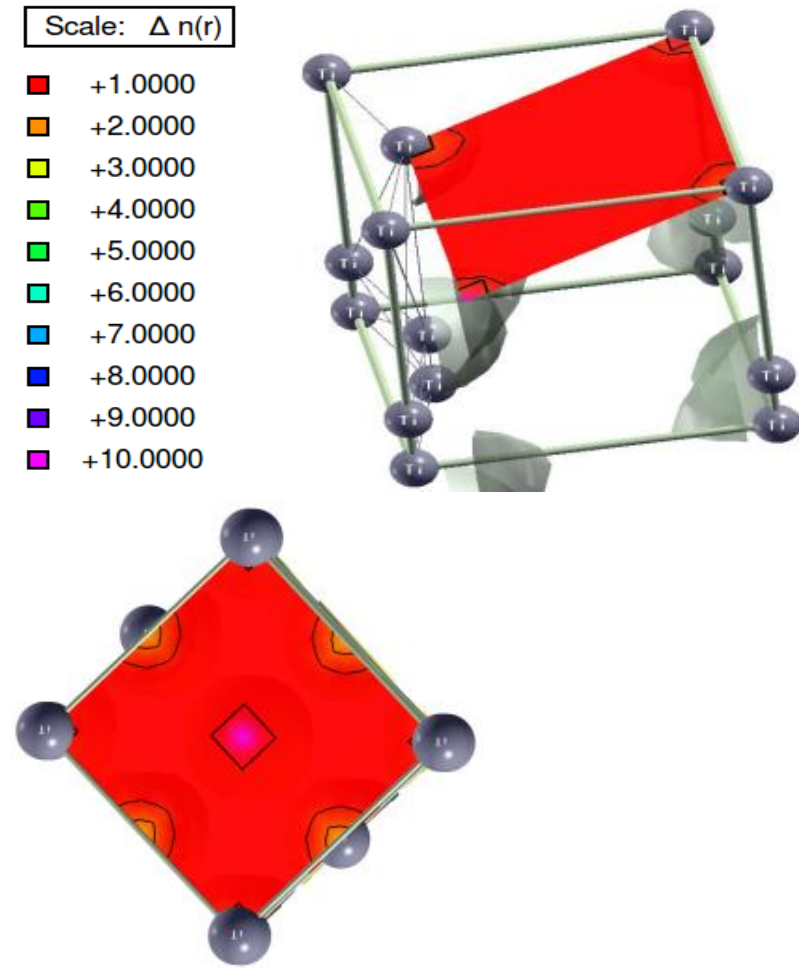

Fig.12: a) Charge Density Scale b) Charge density of $\mathrm{Ti}$ c) Charge density of anatase.

\section{Mxene Anatase nanocomposite}

Mxenes are carbides, nitrides or carbonitries compounds with $M_{n+1} X_{n}$, where $M$ is early transition metal and $\mathrm{X}$ is $\mathrm{C}$ or $\mathrm{N}$ [28]. $\mathrm{n}$ is integers with value 1,2 and $3 . \mathrm{Ti}_{3} \mathrm{C}_{2} \mathrm{~T}_{x}$ of $\sim 600 \pm 200 \mathrm{~nm}$ is stable (very few $1.2 \%$ degradation) for 25 days when kept in argon sealed vials refrigerator $\left(4^{\circ} \mathrm{C}\right)$. Alternately, when the solution is stored at room temperature in air, it is found to be oxidized completely in 15 days. As the process continues, the anatase particles of $\sim 100 \mathrm{~nm}$ along the edges of the flakes and $\sim 2-3 \mathrm{~nm}$ on the basal planes are formed in the process of oxidation in 25 days. This shows that the process is active at the edges. It is also seen that the Mxene film created after vacuum assisted filtration of its colloidal solution significant stability in air. e.g. $\mathrm{Ti}_{3} \mathrm{C}_{2} \mathrm{~T}_{x}$ is stable for 30 days at room temperature in air [29]. It can be true of multilayer powers but not studied mxene fewlayer powder systematically yet.

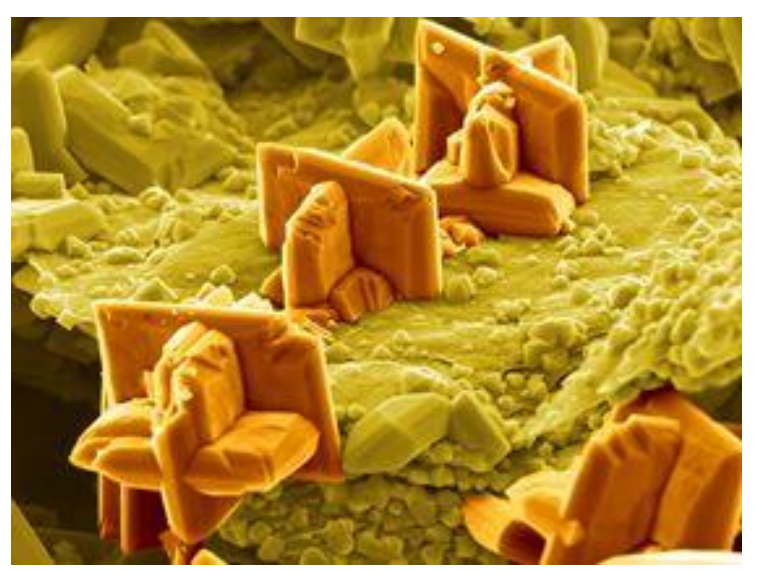

Fig.13: SEM image of Mxene Anatase nanocomposite Retrieved from: Deysher, G. et. al. 2019[30].

The synthesis of mxene oxide is more simpler than that for graphene or carbon allotropes. Carbon supported mxene oxides from hybrid system are used for water treatment [32] lithium-air batteries [33], catalytic converters for exhaust systems [34, 35] and solar cells [36]. e. g. $\mathrm{TiO}_{2}$ particles may act as a photocatalyst for degrading pollution in air and water with the help of activated carbon [32]. The synthesis of these hybrid systems needs multisteps.

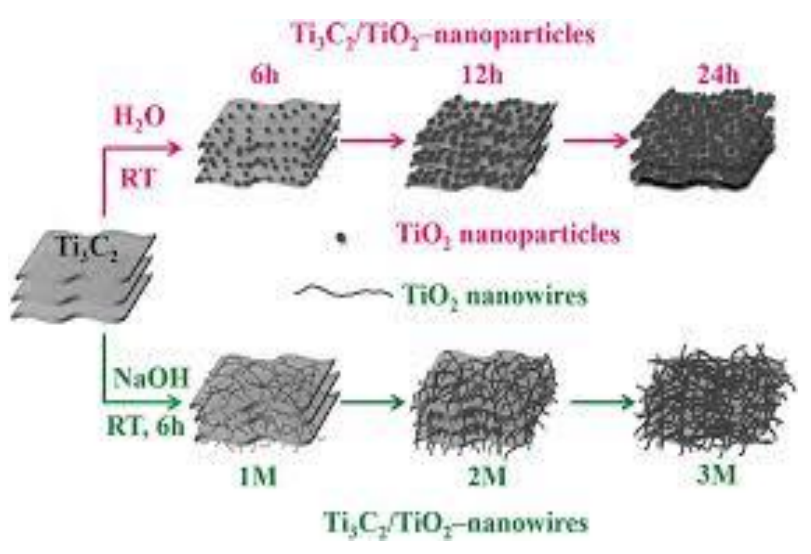

Fig.14: Synthesis of Mxene Anatase nanocomposite Retrieved from: Cao, M. et. al. (2017)[31]. 
$\mathrm{TiO}_{2}$ particles can be developed on graphitic carbon in one step by flash oxidation of MXene powders at $1150^{\circ} \mathrm{C}$ for $30 \mathrm{~s}$ in air for the controlled oxidation of $\mathrm{Ti}_{3} \mathrm{C}_{2} \mathrm{~T}_{x}$ flakes [38]. Similarly, another one-step oxidation methods such as hydrothermal, heating in $\mathrm{CO}_{2}$ [39], and $\mathrm{H}_{2} \mathrm{O}_{2}$-assisted oxidation[38] have also been demonstrated. These different methods can result slightly different properties. They have fewer wrinkles, defects and agglomerates than that in the bottom-up approaches [40].

Anatase when deposited on graphitic carbon by the flash oxidation method can be used as and efficient anode material in lithium ion batteries [38]. Similarly, $\mathrm{TiO}_{2}$ on 2D carbon sheets produced by annealing of $\mathrm{Ti}_{3} \mathrm{C}_{2}$ at $800^{\circ} \mathrm{C}$ for $1 \mathrm{~h}$ in $\mathrm{CO}_{2}$ atmosphere can be used as excellent microwave absorber [40].

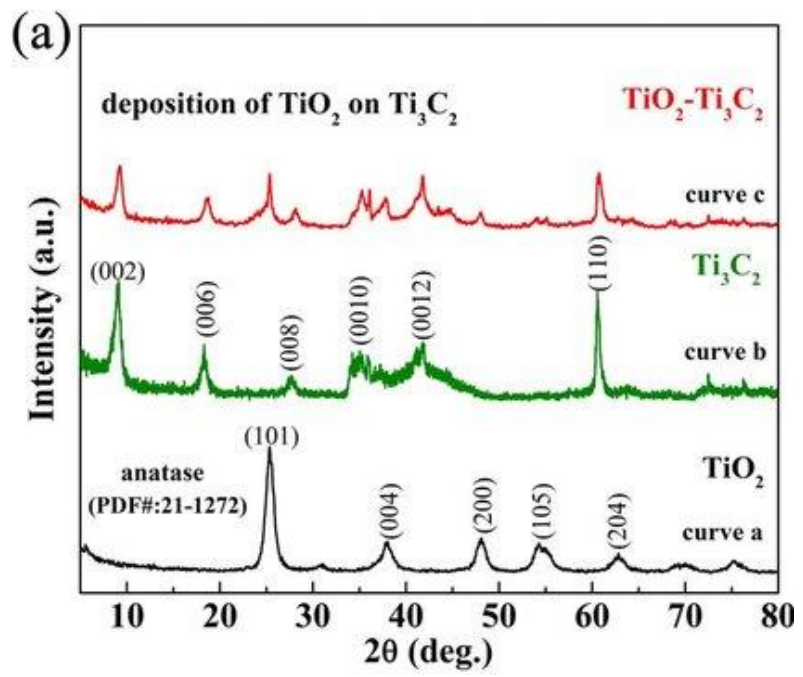

Fig. 15: $\mathrm{XRD}$ plots of $\mathrm{TiO}_{2}-\mathrm{Ti}_{3} \mathrm{C}_{2}, \mathrm{Ti}_{3} \mathrm{C}_{2}$ and $\mathrm{TiO}_{2}$. (Retrieved from: Zhu, J. et. al 2016)[37].

Besides $\mathrm{Ti}_{3} \mathrm{C}_{2}$, several other mxenes including are predicted to be oxidized for several applications high-capacitance electrodes for lithium ion capacitors[41], catalysis, energy storage, photovoltaics and optical sensors[42]. Likewise, Ordered Double Transitional Metal Layered Carbides as topological insulators[43, 44] and $\mathrm{TiO}_{2}-\mathrm{Ti}_{3} \mathrm{C}_{2}$ as nanocomposite for biocompatible material and mediator-free biosensor.

\section{CONCLUSIONS}

The lattice parameter of $\mathrm{Ti}$ at minimum energy is $2.81 \mathrm{~A}^{\mathrm{o}}(5.31 \mathrm{a}$. u). The optimized lattice parameter of anatase using Vegards law is 7.149 a.u. Taking this as base value, the optimized lattice parameter is 7.15 a.u.
The band structure calculation of Titanium shows that only its $d$ orbital electrons have significant contribution in electronic and magnetic properties. As a result, the Titanium is metallic but slightly paramagnetic in nature. Similarly, the band structure calculation of Anatase shows that none of $\mathrm{s}, \mathrm{p}$ and $\mathrm{d}$ orbital electrons have contribution in conduction due to no overlapping between conduction and valance band. So, anatase is semiconductor with wide energy gap of $2.10 \mathrm{eV}$.

The DOS plot of Titanium also shows the large numbers of $\mathrm{d}$ orbital electrons near the Fermi level have the significant role for conduction which ultimately give the corresponding electronic and magnetic property of Titanium. The magnetic moment obtained by integrating difference between the density of states of up spin and down spin of Ti is found to be $2.2 \mu \mathrm{B}$. So, $\mathrm{Ti}$ is metallic but paramagnetic (slight magnetic) in nature. Similarly, from data obtained for density of states of anatase, the magnetic moments of anatase is found to be $0.00000833 \mu \mathrm{B}$, which is negligible at absolute zero. So, anatase has no magnetic moment.

Furthermore, the table of charge density at different orbitals of $\mathrm{Ti}$ shows that only the $\mathrm{d}$ orbitals electrons are transferred and have respective contribution in conduction. The charges are not transferred in any orbital in case of anatase.

Finally, the maximum distribution of the charges occur on the corners of Titanium are contributing in conduction. While, maximum charges occurs at interstitial site of anatase have no contribution in conduction. The calculated values are in good agreement with previous works as mentioned above. Regarding the anatase mxene nanocomposite, it is applicable in diversed field with excellent performance.

\section{ACKNOWLEDGEMENT}

I indebted to Office of Rector, Institute of Science and Technology, Tribhuvan University, Nepal for providing me the grants in support of this work. I am thankful to Prof. Dr. N. P. Adhikari and others for their valuable suggestions and ideas on the subject matter. At last but not the least, I am happy to thank all my family and friends for their cooperation and contributions.

\section{REFERENCES}

[1] Martin, R. M. Electronic structure; Basic Theory and Practical Methods. Cambridge University Press, United Kingdom (1954). 
[2] Hanaor D. A. H., Sorrell C. C. "Review of the anatase to rutile phase transformation" Springer Science \& Business Media, LLC (2010).

[3] Beltran, A., Gracia, L., Andres, J. J Phys Chem B 110:23417 (2006).

[4] Howard, C.J., Sabine, T. M., \& Dickson, F. Acta Crystallographica, B47, 462-468 (1991).

[5] Korringa, J., Phys. Rev. 13, 392 (1947).

[6] Kohn, W. \& Rostoker, J.. Phys. Rev. 94, 1111 (1954).

[7] Skriver, H. L. The LMTO Method Muffin-Tin Orbitals and Electronic Structure. 41, Springerverlag (1984).

[8] Slater, J. C. \& Koster, G. F. Phys. Rev. 50 B. 15:1498-1524 (1954).

[9] Ashcroft, Neil W., Mermin, N. David. Solid state physics. QC176.A83 530.4'1 74-9772 ISBN 0-03083993-9 (College Edition) (1976).

[10] Andersen, O. K., Arcangeli, C., Tank, R.W. , Saha-Dasgupta, T., Krier, G., Jepsen, O. \& Dasgupta, I. Tight-Binding Approach to Computational Materials Science, Eds. L. Colombo, A. Gonis, P. Turchi, MRS Symp. Proc. 491, 3 (1998).

[11] Andersen, O. K., Jepsen, O. \& Krier, G. Lectures in Methods of Electronic Structure Calculations, Eds. V. Kumar, O.K. Andersen, A. Mookerjee, World Sci. Publ. Co., Singapore, p. 63 (1994).

[12] Heldin, L. \& Lundqvist, B. I. J. Phys. C: Solid St. Phys., 4, 2064 (1971).

[13] Mizutani, S. Introduction to the electron theory of Metals. Department of Crystalline Materials Science, Nagoya University, Cambridge Unviersity Press. ISBN 0521583349 (2001).

[14] Hohenberg, P. \& Kohn, W. Phy. Rev. 136, B. 864 (1964).

[15] Kohn, W. \& Sham, L. J. Phys. Rev., 140:A1133 (1965).

[16] Levine, I. N. Quantum Chemistry. Prentice-Hall of India, New Delhi, fifth edition (2006).

[17] Vegard, L. "Die Konstitution der Mischkristalle und die Raumfüllung der Atome". Zeitschrift für Physik. 5 (1): 17-26. Bibcode: 1921ZPhy....5...17V. (1921). doi:10.1007/BF01349680.

[18] Tank, R. W., Jepsen, O. \& Andersen, O. K. The STUTTGART TB-LMTO-ASA program, MaxPlank-Institute fur Festkorperforschung, Heisenbergstr., Germany (2000).

[19] Zohng, W., Overny, G ., \& Tomanek, D. Phys. Rev. B. 47, 1 (1992).

[20] Mookerjee, A., Tarafder, K., Ghosh, S., Sanyal, B., Eriksson, O., \& Chakrabarti, A. Electronic and magnetic properties of disordered $\mathrm{Fe}-\mathrm{Cr}$ alloys using different electronicstructure methods, 20 (2008).

[21] Kaphle, G. C., Ganguly, S., Banerjee, R., Khanal, R., Adhikari, C., Adhikari, N. P. \& Mookerjee, A.: J. Physics: Condens. Matter. 24, 295501 (2012).

[22] Andersen, O. K. Phys. Rev. B. 12:3060 (1975).

[23] Glench, G. ab initio Study on structural changes and phase stability of some transition metals under pressure, Ph. D. Thesis, S.T.H. College, Kanyakumari, Tamil Nadu (2014).

[24] Parajuli, D. First-principles study of Electronic and Magnetic Properties of Ti-Ni Alloy Using TBLMTO-ASA Method. The Journal of University Grants Commission, University Grants Commission, Nepal, Vol. 6, No. 1, ISSN 23508566 (2018).

[25] Wu, S., Xu, M., Wang, S., \& Jia, Yu. Theoretical Study on the Point Defects in N-Doped Anatase $\mathrm{TiO}(2)$, Journal- Korean Physical Society.53 (94): 2292-2298 (2008). DOI: $10.3938 / \mathrm{jkps}$

[26] Kittel, C. Introduction to solid state physics. Wiley Publication, seventh edition (2009).

[27] García, E.G.P et. al. Tutorial: GNU Plot for DOS of Anatase- a computational work using VASP, Laboratory of molecular and applied atomic physics, Metropolitan Autonomous University Azcapotzalco, Mexico (2018). http://molphys.org/gnuplot_tutorial/dos_plot.html

[28] Gogosti, Y.: "Nanomaterials in electrical energy storage applications" HDIAC Journal, 3(3) (2016).

[29] C.J. Zhang, et al.Oxidation stability of colloidal two-dimensional titanium carbides (Mxenes) Chem. Mater., 29 (11), 4848 (2017).

[30] Deysher, G., et. al. Oxidized 2D titanium carbide Mxene, Material Today, Volume 21, Issue 10, Page 1064-1065 (2019).

[31] Cao, M. et. al.. Room Temperature Oxidation of Ti3C2MXene for Supercapacitor Electrodes, Journal of The Electrochemical Society, 164(14) A3933-A3942 (2017).

[32] G. L. Puma, et al. Preparation of titanium dioxide photocatalyst loaded onto activated carbon support using chemical vapor deposition: a review paper,J. Hazard. Mater., 157(2-3), 209219 (2008)

[33] I. Roche, et al. Carbon-supported manganese oxide nanoparticles as electrocatalysts for the oxygen reduction reaction (ORR) in alkaline medium: physical characterizations and ORR mechanism, J. Phys. Chem. C,111(3), pp.14341443 (2007).

[34] Z. Zhu, et al.A novel carbon-supported vanadium oxide catalyst for $\mathrm{NO}$ reduction with $\mathrm{NH}_{3}$ at low 
temperatures, Appl. Catal. B: Environ.,23 (4), pp.L229-L233 (1999).

[35] C. Heisig, W. Zhang, S.T. Oyama Decomposition of ozone using carbon-supported metal oxide catalysts, Appl. Catal. B: Environ., 14 (1-2), pp.117-129 (1997).

[36] C. Dall'Agnese, et al., Oxidized $\mathrm{Ti}_{3} \mathrm{C}_{2}$ MXene nanosheets for dye-sensitized solar cells, New J. Chem., 42 (20) pp. 16446-16450 (2018).

[37] Zhu, J. et. al.. Composites of $\mathrm{TiO}_{2}$ Nanoparticles Deposited on $\mathrm{Ti}_{3} \mathrm{C}_{2}$ MXene Nanosheets with Enhanced Electrochemical Performance, Journal of The Electrochemical Society 163(5):A785A791 (2016).

[38] M. Naguib, et al. One-step synthesis of nanocrystalline transition metal oxides on thin sheets of disordered graphitic carbon by oxidation of Mxenes Chem. Commun.,50(56), pp.74207423 (2014).

[39] B. Ahmed, et al. $\mathrm{H}_{2} \mathrm{O}_{2}$ assisted room temperature oxidation of $\mathrm{Ti}_{2} \mathrm{C}$ MXene for Li-ion battery anodes Nanoscale,8 (14), pp.7580-7587 (2016).
[40] M. Han, et al. Laminated and two-dimensional carbon-supported microwave absorbers derived from Mxenes ACS Appl. Mater. Interfaces, 9 (23), pp. 20038-20045 (2017).

[41] C. Zhang, et al. Synthesis and charge storage properties of hierarchical niobium pentoxide/carbon/niobium carbide (MXene) hybrid materials Chem. Mater., 28 (11), pp. 39373943 (2016).

[42] S. Chertopalov, et al. Environment-sensitive photoresponse of spontaneously partially oxidized $\mathrm{Ti}_{3} \mathrm{C}_{2} \mathrm{MXene}$ thin films ACS Nano., 12 (6), pp. 6109-6116 (2018).

[43] Khazaei, M., et. al. Topological insulators in the ordered double transition metals $M_{2}^{\prime}{ }_{2}{ }^{\prime \prime} \mathrm{C}_{2}$ Mxenes $\left(M^{\prime}=M o, W ; M^{\prime \prime}=T i, \quad Z r, \quad H f\right)$, PHYSICAL REVIEW B 94, 125152; 2469-9950 (2016).

[44] Anasori, B. et. al. Two-Dimensional, Ordered, Double Transition Metals Carbides (Mxenes), ACS Nano, VOL. 9, NO. 10, 9507-9516 (2015). 